\title{
Identification of 7 Movements of the Human Hand Using SEMG - $360^{\circ}$ on the Forearm
}

\section{Identificación de 7 Movimientos de la Mano Humana Utilizando sEMG - 360 en el Antebrazo}

\author{
A. Ibarra-Fuentes, E. Morales-Sánchez \\ Instituto Politécnico Nacional. CICATA-IPN Unidad Querétaro. México.
}

\begin{abstract}
This document shows the identification of 7 gestures (movements) of the human hand from sEMG $-360^{\circ}$ signals on the forearm. sEMG $-360^{\circ}$ is the sEMG measurement through 8 channels every $45^{\circ}$ making a total of $360^{\circ}$. When making a hand gesture, there will be 8 independent sEMG signals that will be used to identify the gesture. The 7 gestures to identify are: relaxed hand (closed), open hand (fingers extended), flexion and extension of the little finger, the ring finger, the middle finger, the index finger, and the thumb separately. One hundred samples for each gesture were captured and 3 feature extraction methods were applied in the time domain: mean absolute value (MAV), root mean square value (RMS) and area under the curve (AUC). A vector support machine (SVM) classifier was applied to each extractor. The gestures were identified and the percentage of accuracy in the identification was calculated for each extractor + SVM classifier using the confusion matrix method and including the 8 channels for each gesture. An accuracy of $99.52 \%$ was achieved for the identification of the 7 gestures applying sEMG - $360^{\circ}$.
\end{abstract}




\section{RESUMEN}

Este documento muestra la identificación de 7 gestos (movimientos) de la mano humana a partir de sEMG - 360 ${ }^{\circ}$ en el antebrazo. sEMG-360 es la medición de sEMG por medio de 8 canales cada $45^{\circ}$ haciendo un total de $360^{\circ}$. Al realizar un gesto de la mano se tendrán 8 señales sEMG independientes que se utilizarán para hacer la identificación del movimiento. Los 7 gestos a identificar fueron: mano relajada (cerrada), mano abierta (dedos extendidos), flexión y extensión del dedo meñique, del dedo anular, del dedo medio, del dedo índice y del dedo pulgar por separado. Se capturaron 100 muestras de cada gesto y se aplicaron 3 métodos de extracción de características en el dominio del tiempo: el valor medio absoluto (MAV), valor de la raíz cuadrática media (RMS) y el valor del área bajo la curva (AUC), después se aplicó un clasificador de máquina de soporte vectorial (SVM) a cada método de extracción. Se identificaron los movimientos y se calculó el porcentaje de exactitud en la identificación para cada extractor + clasificador SVM utilizando el método de la matriz de confusión e incluyendo los 8 canales para cada gesto. Se logró un 99.52\% de exactitud en la identificación de los gestos de la mano humana aplicando sEMG - 360 .

PALABRAS CLAVE: Electromyography, gestures, classifier

\section{Corresponding author}

TO: Eduardo Morales Sánchez

INSTITUTION: Instituto Politécnico Nacional

ADDRESS: Av. Luis Enrique Erro S/N, Unidad Profesional

Adolfo López Mateos, Col. Zacatenco, Alc. Gustavo A.

Madero, C. P. 07738, CDMX, México

E-MAIL: emoraless@ipn.mx

\section{Received:}

18 June 2021

\section{Accepted:}

4 November 2021 


\section{INTRODUCTION}

Electromyography is a technique that measures the bioelectrical signals that muscles generate when the body performs an action; these signals have been used for the performance analysis of athletes, for the remote control of highly complex mechanical and electronic systems in movement, to measure development in rehabilitation, among other applications. sEMG signals are acquired by means of surface electrodes.

The literature reports the use of 1, 2 and up to 3 electrode arrays using the RLD (Right Leg Driven) configuration. The commercial device called Myo Armband, Thalmic labs ${ }^{[1]}$, is a wireless electronic bracelet consisting of 8 channels ( 8 arrays of 3 mini dry-surface electrodes). When this bracelet of 8 arrangements of 3 superficial electrodes is positioned on the forearm, you have a complete overview of the sEMG signals of the forearm muscles when the hand performs any movement. This way of measuring EMG around a human extremity is called SEMG-360.

In recent years, different researches have considered sEMG signals to identify the intention of movement of the human hand and to be able to reproduce them in a robotic hand. These signals are acquired when different gestures (movements) are made and then they are processed to acquire the most important information of the signal and once processed they can be entered into a classifier in order to identify the movements made by the user.

Tavakoli et al. ${ }^{[2]}$ reports the sEMG signals of the forearm when certain movements are performed: open hand, closed hand, hand at rest and wrist flexion, these are acquired with the use of 3 superficial electrodes, then an extraction of characteristics is made in the time domain to the signals, where the mean value (MV) of each of them is obtained, then an SVM classifier is used to predict the movements, where they obtained an accuracy of $90 \%$.
Another study presented by Shi et al. ${ }^{[3]}$, where superficial electrodes are used to acquire the SEMG signals from the forearm when performing 4 gestures such as: closed hand, extended index finger, extended thumb, and the 4 extended fingers together. An extraction of characteristics in the time domain was applied, where the main descriptors were: the MAV, the zero crossing (ZC), the slope sign change (SSC) and the waveform length (WL), these characteristics were used in the nearest neighbor classifier (KNN) where they obtained an accuracy of 94\%.

In the study presented by Krishnan et al. ${ }^{[4]}$, the Myo Armband device is used to acquire EMG signals by performing 5 movements of the human hand. The author used some feature extraction methods in the time domain as the simple square integral (SSI), the maximum value and the minimum value, the average frequency and the average potential; these methods were used in an SVM classifier where they obtained an identification accuracy percentage of $92.4 \%$ for one user and $84.27 \%$ for another user.

In Mukhopadhyay et al. ${ }^{[5]}, 8$ arrays of 3 electrodes are used to predict 8 classes of hand movement: wrist flexion, wrist extension, wrist pronation, wrist supination, force grip, pinch grip, open hand, and rest. Powerspectral descriptors were used to extract the characteristics of the sEMG signals. Subsequently, they used a deep neural network (DNN) as a classifier, obtaining 98.88\% accuracy.

In the study presented by Sanchez et al. ${ }^{[6]}$, the author obtained sEMG signals by using the Myo Armband bracelet to predict 8 hand gestures to reproduce on a robotic hand. They used an extraction of characteristics in the time domain as MAV, the RMS, the WL, the mean amplitude change (AAC), the integrated EMG value (IEMG) and the absolute standard deviation (DASDV); these data were used in an extended associative memory (EAM) classifier and obtained an accuracy of $94.83 \%$ when using the MAV and RMS extractors. 
Table 1 shows a summary of the previous literature that includes different characteristics such as the number of electrodes, their position, number of gestures, type of feature extraction method, and the identification accuracy.

TABLE 1. Summary of the previous works in the state of the art about EMG and classification of gestures.

\begin{tabular}{|c|c|c|c|c|c|}
\hline 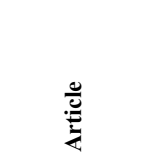 & 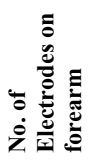 & 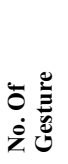 & 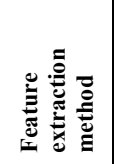 & 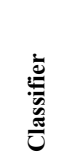 & 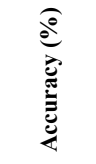 \\
\hline $\begin{array}{l}\text { Tavakoli } \\
\text { et al }^{[2]}\end{array}$ & 1 & 4 & MV & SVM & 90 \\
\hline $\begin{array}{l}\text { Shi } \\
\text { et al }{ }^{[3]}\end{array}$ & 2 & 4 & $\begin{array}{l}\text { MAV, } \\
\text { ZC, SSC } \\
\text { and WL }\end{array}$ & KNN & 94 \\
\hline $\begin{array}{l}\text { Krishnan } \\
\text { et al }^{[4]}\end{array}$ & 8 & 5 & $\begin{array}{l}\text { SSI, Max, } \\
\text { Min, MF } \\
\text { and MP }\end{array}$ & SVM & 92.4-84.27 \\
\hline $\begin{array}{l}\text { Mukhopadhyay } \\
\text { et al }^{[5]}\end{array}$ & 7 & 8 & fTDD & $\begin{array}{c}\text { DNN } \\
\text { SVMK } \\
\text { NNRF } \\
\text { DT }\end{array}$ & $\begin{array}{l}98.66, \\
90.64, \\
91.78, \\
88.36\end{array}$ \\
\hline $\begin{array}{l}\text { Sanchez } \\
\text { et al }^{[6]}\end{array}$ & 8 & 8 & \begin{tabular}{|c|} 
IEMG, \\
MAV, VAR, \\
RMS, \\
DASDV \\
AAC \\
and WL \\
\end{tabular} & EAM & 95.83 \\
\hline
\end{tabular}

From the previous review it can be seen that there are different articles on the identification of some hand gestures using different extractors and classifiers [7] with 1, 2, 4, and 8 array electrodes but with different identification accuracy percentage. Due to the random nature of sEMG signals, it is of the researchers' interest to improve the identification accuracy of human hand gestures using EMG signals.

The present work proposes to improve the gesture accuracy identification using only sEMG-360 array and keeping the same feature extraction methods (MAV, RMS and AUC) in the time domain and the SVM classifier to identify 7 gestures of the human hand.

\section{MATERIALS AND METHODS}

This section shows the methodology used to extract the characteristics of the sEMG-360 signals acquired (in the 8 channels at $45^{\circ}$ around the forearm) corre- sponding to 7 movements of the human hand: open hand (5 fingers extended), closed hand (5 fingers flexed), and 5 flexion-extension movements for each finger individually, as well as to explain the method used to evaluate the performance of the identification.

\section{Myo Armband bracelet}

It is an electronic device developed by the Thalmic Lab company as illustrated in Figure $1^{[1]}$. It is a bracelet that can be placed on the forearm to record the sEMG activity that is generated by the movement of the muscles. This device has 8 arrays of 3 dry-surface electrodes to monitor sEMG, it combines an accelerometer, a gyroscope, a magnetometer, an ARM Cortex M4 processor, indicator LEDs, motor vibrators, Bluetooth communication and a rechargeable power supply.

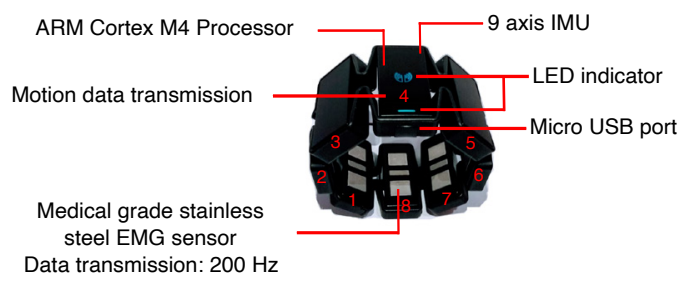

FIGURE 1. Characteristics of the Myo Armband.

The sampling frequency of the Myo Armband bracelet is $200 \mathrm{~Hz}$. The total sample rate of the Myo Armband is 200 $\mathrm{Hz}$, which gives us a sample time as shown in Equation 1:

$$
T_{\text {sampling }}=1 / 200 \mathrm{~Hz}=5 \mathrm{~ms}
$$

Where $T_{\text {sampling }}$ is the sampling time, $\mathrm{Hz}$ is the sample rate and $m s$ equals milliseconds.

This indicates that every $5 \mathrm{~ms}$, the EMG signals of the 8 channels are acquired in $360^{\circ}$ configuration of the Myo Armband bracelet.

\section{Acquisition of EMG signals}

The Myo Armband device was used to acquire the 8 sEMG signals (8 channels) for each flexion-extension movement of the fingers of the human hand, the 
extended hand position (open) and the relaxed hand (closed). By means of Bluetooth (integrated in the device) and using the Python software, each of the 8 channels of each of the established movements were captured and saved on a PC for later analysis.

A window of 400 data was generated as shown in Figure 2, where the sEMG signals of the 8 channels are graphed. The movement developed in Figure 2 corresponds to the flexion-extension movement of the middle finger. A segment of 400 samples represents 2 seconds of the signal, though the gesture took $1 \mathrm{sec}-$ ond, the rest is the time interval before and after the gesture.

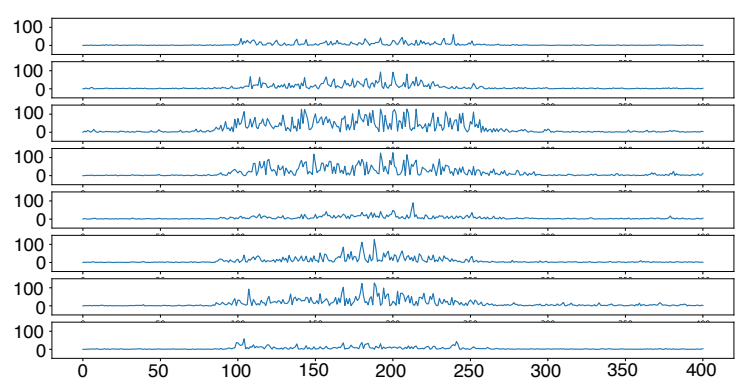

FIGURE 2. sEMG signals of the 8 channels, corresponding to the flexion-extension movement of the middle finger.

Once the sEMG signals were acquired for the preset gestures, feature extraction methods of the 8 channels were performed for the 7 motions. These feature extractions were performed in the time domain. It was proposed to implement 3 types of extractors, which are the most reported in the literature: the mean absolute value method (MAV) ${ }^{[8]}$, the root mean square (RMS) method ${ }^{[9]}$ and the area method under the curve (AUC) ${ }^{[6]}$.

In the MAV method, the mean value of the sEMG signal is calculated. The formula to calculate it is shown in Equation 2.

$$
\mathrm{MAV}=\frac{1}{N} \sum_{i=1}^{N}\left|X_{i}\right|
$$

Where $N$ represents the number of data contained in the sample and $X i$ the data contained in said sample.

The RMS method obtains the root mean square value of the sEMG signal. The mathematical expression is shown in Equation 3.

$$
\mathrm{RMS}=\sqrt{\frac{1}{N} \sum_{i=1}^{N} X_{i}^{2}}
$$

Where $N$ represents the number of data contained in the sample and $X i$ the data contained in said sample.

The area method consists of applying the trapezoid rule as an approximation of the definite integral by adding the areas of the trapezoids that make up the sEMG signal. The formula for calculating the area under the curve is shown in Equation 4.

$$
T_{N}(f)=\frac{\Delta_{x}}{2} \sum_{i=1}^{N}\left(f\left(x_{i}\right)+f\left(x_{i}-1\right)\right)
$$

Where $\Delta x=(b-a) / N$ is the length of the subintervals and $X i=a+i \Delta x$. The values of $a$ and $b$ belong to the interval where the integral will be evaluated.

\section{Classifier}

Once the feature extraction has been carried out using each of the proposed extraction methods, a classifier is required to be applied to separate the information and identify each movement. For this study, the support vector machine classifier (SVM) is used, where 100 data of each of the predefined gestures will be used. The data used for SVM can be more or less than one hundred, according to literature, one hundred is a representative number when statistics is applied [10] [11] [12]; the SVM classifier is an algorithm that can determine a plane that separates the acquired data set into several sets (vectors). For a new set, the similarity of the vector is determined, and it is classified within the set associated with that vector. 
SVM algorithms use a set of mathematical functions called kernels. There are different types of kernel functions, such as: linear, polynomial, radial basis function (RBF) among others. Depending on the type of kernel, an accurate or inaccurate classification of the data is achieved.

The kernel functions are represented as:

- Linear: $z=y * x+b$

- Polynomial: $z=\left(y^{*} x+b\right)^{n}$

- RBF: $z=\exp \left(-\gamma\|x-y\|^{2}\right)$

Where $x$ and $y$ are the data that is entered into the classifier, $b$ is a parameter to improve performance and $n$ is the degree of the polynomial, $\gamma$ defines how much influence a single training example has.

In this work, the 3 types of kernels were applied for the SVM in order to determine which kernel maximizes the percentage of accuracy in the identification of movements.

\section{Confusion matrix}

In order to calculate the percentage of accuracy that exists during an identification process, the confusion matrix is used.

In the confusion matrix, each column represents the number of predictions for each class, and the rows represent the instances of the real class, so it is possible to observe the successes and errors of the model during the identification of the movement ${ }^{[13]}$. Figure 2 shows a confusion matrix for 4 classes: A, B, C and D.

To calculate the accuracy value (AC), Equation $5^{[14]}$ is used.

$$
A C=\frac{T}{T+F A B+F A C+F A D+F B A+\ldots+F D C+F C D}
$$

Where $T=V A+V B+V C+V D ; V A, V B, V C$ and $V D$ are the numbers where the classes are identified as true. $F A B$....FCD is the number in which a class is classified in another class. With100\% accuracy, all the values for $F A B$... FCD are 0.

TABLE 2. Confusion matrix for 4 classes.

\begin{tabular}{|c|c|c|c|c|c|c|}
\hline & & \multicolumn{5}{|c|}{ Actual Class } \\
\hline & & A & B & $\mathrm{C}$ & $\mathrm{D}$ & $\ldots$ \\
\hline \multirow{5}{*}{ 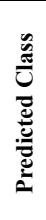 } & A & VA & FAB & FAC & FAD & $\ldots$ \\
\hline & B & FBA & $\mathrm{VB}$ & FBC & FBD & $\ldots$ \\
\hline & $\mathrm{C}$ & FCA & FCB & $\mathrm{VC}$ & FCD & $\ldots$ \\
\hline & $\mathrm{D}$ & FDA & FDB & FDC & $\mathrm{VD}$ & $\ldots$ \\
\hline & $\ldots$ & $\ldots$ & $\ldots$ & $\ldots$ & $\ldots$ & ... \\
\hline
\end{tabular}

\section{RESULTS AND DISCUSSION}

Since each gesture has 8 associated sEMG channels, it is ideal to find the relationship of the 8 channels with the gesture. One technique used is to graph the data in an eight-dimensional space (number of channels). To simplify and show graphically this relationship, Figure 3 shows the corresponding three-dimensional space data for channels 1, 2 and 6 of each of the 7 movements using the MAV extractor (different colors). It is observed that the classes are separated since they are in different ranges within the three-dimensional space. It is observed that the open hand and closed hand gestures are naturally separated from the gestures of moving a single finger. It is also observed that the little finger, middle finger and ring finger are the gestures that least allow classification because they overlap each other.

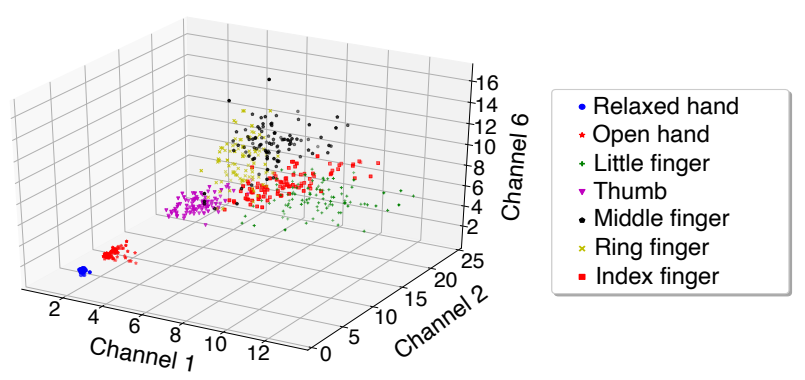

FIGURE 3. MAV characteristics of channels 1, 2 and 6. 
The behavior was calculated for the CUA and RMS extractors. There were no significant differences between them.

Once the MAV, AUC and RMS feature extractors were applied to the SEMG signals, the SVM classifier was applied. Figure 4 shows the classification carried out by the SVM with a linear kernel of the data obtained from the MAV for channels 1 and 6. It is observed that there are some data that were misclassified since they are in the region of a different class.

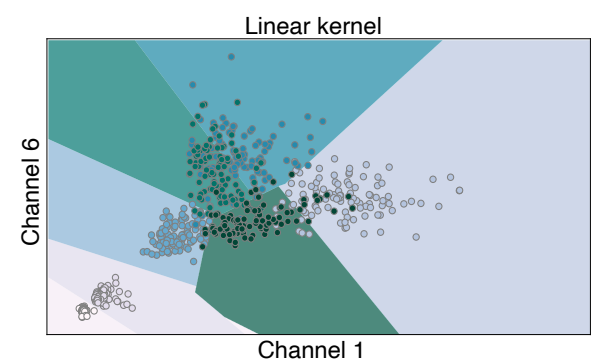

FIGURE 4. Classification of data obtained from

the MAV when using a linear kernel in the SVM.

Considering the classification obtained from the classes with 100 samples for each gesture, the corresponding confusion matrix was obtained (Table 3). $70 \%$ of the data was used for training and the remaining 30\% for testing. Table 2 was obtained for a linear kernel SVM.

TABLE 3. Confusion matrix for the identification of the 7 movements. Shown for SVM with RMS as feature extraction method.

\begin{tabular}{|c|c|c|c|c|c|c|c|}
\hline & $(\mathbf{1})$ & $\mathbf{( 2 )}$ & $\mathbf{( 3 )}$ & $\mathbf{( 4 )}$ & $\mathbf{( 5 )}$ & $\mathbf{( 6 )}$ & $\mathbf{( 7 )}$ \\
\hline $\mathbf{( 1 )}$ & 30 & 0 & 0 & 0 & 0 & 0 & 0 \\
\hline $\mathbf{( 2 )}$ & 0 & 30 & 0 & 0 & 0 & 0 & 0 \\
\hline $\mathbf{( 3 )}$ & 0 & 0 & 30 & 0 & 0 & 0 & 0 \\
\hline $\mathbf{( 4 )}$ & 0 & 0 & 0 & 30 & 0 & 0 & 0 \\
\hline $\mathbf{( 5 )}$ & 0 & 0 & 0 & 0 & 30 & 0 & 0 \\
\hline $\mathbf{( 6 )}$ & 0 & 0 & 0 & 0 & 0 & 30 & 0 \\
\hline $\mathbf{( 7 )}$ & 0 & 0 & 0 & 0 & 3 & 0 & 27 \\
\hline
\end{tabular}

In Table 3, number (1) refers to the gesture of the closed hand, number (2) the open hand, number (3) is the flexion-extension (FE) of the little finger, number (4) is for FE of the thumb, number (5) for FE of the middle finger, number (6) for $\mathrm{FE}$ of the ring finger and finally number (7) refers to FE of the index finger. In the same way, the black-colored diagonal values show the successes that a class has in respect to itself, and the other values that are outside the diagonal, refer to how many times the classifier predicted a value of a class that does not belong to the assigned one.

Table 4 shows the percentage of accuracy in the identification of the 7 gestures. It is shown that the RMS + SVM with the linear kernel, RMS + SVM with rbf kernel and AUC + SVM with rbf kernel obtained the highest percentage of accuracy in the identification with $99.52 \%$.

\section{TABLE 4. Percentage of accuracy in the identification} of gesture under different extractors and kernels.

\begin{tabular}{|c|c|c|c|}
\hline Article & Extractor & SVM kernel & Accuracy \% \\
\hline \multirow{9}{*}{ This work } & MAV & Linear & 98.57 \\
\hline & MAV & Polynomial & 99.04 \\
\hline & MAV & RBF & 98.09 \\
\hline & RMS & Linear & 99.52 \\
\hline & RMS & Polynomial & 99.04 \\
\hline & RMS & RBF & 99.52 \\
\hline & AUC & Linear & 98.57 \\
\hline & AUC & Polynomial & 99.04 \\
\hline & AUC & RBF & 99.52 \\
\hline $\begin{array}{l}\text { Tavakoli } \\
\text { et al }^{[2]}\end{array}$ & MV & Gaussian & 90.00 \\
\hline $\begin{array}{l}\text { Krishnan } \\
\text { et al }^{[4]}\end{array}$ & $\begin{array}{l}\text { SSI, Max, Min, } \\
\text { MF and MP }\end{array}$ & Linear & 92.4 and 84.27 \\
\hline $\begin{array}{l}\text { Mukhopadhyay et } \\
\text { al }^{[5]}\end{array}$ & fTDD & Linear & 98.66 \\
\hline
\end{tabular}

The RBF hyperparameters used were $\mathrm{C}=10000.0$ and Gamma $=1 \mathrm{e}-05$ for MAV feature, for RMS were used C $=10000000$ and Gamma $=1 \mathrm{e}-09$, and for the AUC the value of $\mathrm{C}=100.0$ and $\mathrm{Gamma}=1 \mathrm{e}-09$. The Polynomial parameter degrees used for MAV, RMS and AUC were the value of 2 . 
As shown in Table 4, the best combination of extractor + classifier was that of RMS + SVM with linear kernel also the RMS +SVM and AUC + SVM with the RBF kernel. In a general analysis, it can be observed that there was no significant difference between the feature extraction methods.

According to Table 4, in all the cases we have a high-percentage of identification accuracy, this means that realistically, the sEMG-360 could improve the accuracy. This result was expected because having 8 channels for each gesture to identify is a real advantage comparing with literature (Table 1). In the introduction, Mukhopadhyay et al. ${ }^{[5]}$ using 8 arrays of 3 electrodes to predict 8 gestures and using a deep neural network (DNN) as a classifier, 98.98\% accuracy was obtained. This high accuracy may be because the author used 8 electrodes, as we did, as well as a DNN classifier. In our case, identification accuracy is in the range of 98.5-99.5\% in all the cases. In a different way, it is observed that a second parameter for increased identification accuracy is the classifier SVM. And a third parameter is the feature extraction method. According with our results, RMS is the better extraction method compared to MAV and AUC. At last, from a computational point of view, RMS + SVM implementation is very easy if compared to the DNN classifier.

Therefore, this research shows that s-EMG-360 improves the accuracy of identification though it is used with the common feature extraction method (RMS, MAV, AUC) and SVM classifier.

\section{CONCLUSIONS}

The results show that the RMS extractor in conjunction with the SVM classifier is the combination with the best percentage of accuracy (99.52\%) in the identification of the 7 proposed movements of the fingers of the human hand.
It is shown that using a Myo Armband device is a good option for the implementation of the sEMG-360 method.

The sEMG-360 method improves the identification accuracy of human hand gestures. The SVM classifier was the second principal parameter to improve identification accuracy.

It is concluded that the feature extraction methods in the time domain (MAV, AUC and RMS) give the same result for identification accuracy, with RMS having a slight advantage.

At last, we concluded that sEMG-360 around the forearm is a powerful technique because it adds 8 sEMG to the processing data for gesture identification.

\section{AUTHOR CONTRIBUTIONS}

E.M.S. conceptualized the project, obtained funding for the research, and developed the methodology implemented in the project. A.I.F. developed the software used in the classification of gestures and movements as well as the one used for the storage of the sEMG signals, he also oversaw the data curation and research for the EMG signals and carried out the validation of the movements and the formal analyses of data. Both authors work equally in the writing and editing of the different versions of the manuscript until complete the final version.

\section{ACKNOWLEDGMENTS}

Author Adrian Ibarra thanks the "Consejo Nacional de Ciencia y Tecnología” (CONACYT-Mexico) for his Master post-graduate scholarship. This research was funded by Instituto Politécnico Nacional (IPN) through SIP20196083, SIP20200568 and SIP20211175 projects. 


\section{REFERENCES}

[1] Visconti P, Gaetani F, Zappatore GA, Primiceri P. Technical Features and Functionalities of Myo Armband: An Overview on Related Literature and Advanced Applications of Myoelectric Armbands Mainly Focused on Arm Prostheses. Int J Smart Sens Intell Syst [Internet]. 2018;11(1):1-25. Available from: https://doi.org/10.21307/ijssis-2018-005

[2] Tavakoli M, Benussi C, Lourenco JL. Single channel surface EMG control of advanced prosthetic hands: A simple, low cost and efficient approach. Expert Syst Appl [Internet]. 2017;79:322-322. Available from: http://dx.doi.org/10.1016/i.eswa.2017.03.012

[3] Shi W-T, Lyu Z-J, Tang S-T, Chia T-L, et al. A bionic hand controlled by hand gesture recognition based on surface EMG signals: A preliminary study. Biocybern Biomed Eng [Internet]. 2018;38(1):126-135. Available from: http://dx.doi.org/10.1016/j.bbe.2017.11.001

[4] Krishnan KS, Saha A, Ramachandran S, Kumar S. Recognition of human arm gestures using Myo armband for the game of hand cricket. 2017 IEEE 5th Int Symp Robot Intell Sensors (IRIS) [Internet]. Ottawa: IEEE; 2017:389-394. Available from: https://doi.org/10.1109/IRIS.2017.8250154

[5] Mukhopadhyay AK, Samui S. An experimental study on upper limb position invariant EMG signal classification based on deep neural network. Biomed Signal Process Control [Internet]. 2020;55:101669. Available from: https://doi.org/10.1016/j.bspc.2019.101669

[6] Sánchez Velasco LE, Arias Montiel M, Guzmán Ramírez E, Lugo E. A low-cost EMG-controlled anthropomorphic robotic hand for power and precision grasp. Biocybern Biomed Eng [Internet].

2020;40(1):221-237. Available from: https://doi.org/10.1016/j.bbe.2019.10.002

[7] Rascón-Madrigal LH, Sinecio-Sidrian MA, Mejía-Muñoz JM, DíazRomán JD, Canales-Valdiviezo I, Botello-Arredondo AI. Estimación en la Intención de Agarres: Cilíndrico, Esférico y Gancho Utilizando Redes Neuronales Profundas. Rev Mex Ing Biomed [Internet] 2020;41(1):117-127. Available from: https://dx.doi.org/10.17488/RIIB.41.1.9
[8] Veer K, Sharma T. Extraction and Analysis of above elbow SEMG for Pattern classification. J Med Eng Technol [Internet]. 2016;40(4):149-154. Available from: https://doi.org/10.3109/03091902.2016.1153739

[9] Phinyomark A, Phukpattaranont P, Limsakul C. Feature reduction and selection for EMG signal classification. Expert Syst Appl [Internet]. 2012;39(8):7420-7431. Available from: http://dx.doi.org/10.1016/j.eswa.2012.01.102

[10] Resendiz Trejo J. Las máquinas de vectores de soporte para identificación en línea. México [Master's thesis]. [Honolulu]: Centro de Investigaciòn y de Estudios Avanzados del Instituto Politécnico Nacional, 2006. 84p

[11] Alkan A, Günay M. Identification of EMG signals using discriminant analysis and SVM classifier. Expert Syst Appl [Internet]. 2012;39(1):44-47. Available from: http://dx.doi.org/10.1016/j.eswa.2011.06.043

[12] Hassan HF, Abou-Loukh SJ, Ibraheem IK. Teleoperated robotic arm movement using electromyography signal with wearable Myo armband. J King Saud Univ Eng Sci [Internet]. 2020;32(6):378-387. Available from: https://doi.org/10.1016/j.jksues.2019.05.001

[13] Ting KM. Confusion Matrix. In: Sammut C, Webb GI, editors. Ency Mach Learn [Internet]. Boston, MA: Springer US; 2010. 209p. Available from: https://doi.org/10.1007/978-0-387-30164-8 157

[14] Shamsudin NF, Basiron H, Saaya Z, Rahman AFNA, et al. Sentiment classification of unstructured data using lexical based techniques. J Teknol [Internet]. 2015;77(18):113-120. Available from: https://doi.org/10.11113/jt.v77.6497 